Boletín de la Sociedad Zoológica del Uruguay, 2021

Vol. 30 (2): e30.2.5

ISSN 2393-6940

https://journal.szu.org.uy

DOI: https://doi.org/10.26462/30.2.5

\title{
LA DIVERSIDAD ESCONDIDA: INVERTEBRADOS DE CHARCOS TEMPORALES EN BARRA GRANDE URUGUAY
}

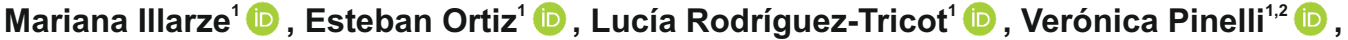 \\ Juan Manuel Piñeiro-Guerra ${ }^{2}$ (D) , Lucía Sosa-Panzera ${ }^{1}$, Matías Zarucki ${ }^{3}$ (D) , Daniel Hernández ${ }^{3}$ (D), \\ Lucía Ziegler $^{1}$ (D), Mauro Berazategui' ${ }^{1}$, , Ana Inés Borthagaray' (D) , Marcelo Loureiro ${ }^{4}$ (D) , \\ Gabriel Laufer ${ }^{5}$ (D) Matías Arim ${ }^{1 *}$ (D). \\ ${ }^{1}$ Centro Universitario Regional del Este (CURE), Universidad de la República, Uruguay. \\ ${ }^{2}$ Departamento de Sistemas Ambientales, Facultad de Agronomía, Universidad de la República, Uruguay. \\ ${ }^{3}$ Polo Educativo Tecnológico Arrayanes, CETP/UTU, Uruguay \\ ${ }^{4}$ Departamento de Ecología y Evolución, Facultad de Ciencias, Universidad de la República, Uruguay. \\ ${ }^{5}$ Museo Nacional de Historia Natural, MEC; Vida Silvestre Uruguay, Uruguay.
}

*Autor para correspondencia: matiasarim@gmail.com

Fecha de recepción: 18 de junio de 2021

Fecha de aceptación: 17 de setiembre de 2021

\section{RESUMEN}

Los charcos temporales de Uruguay y los invertebrados que en estos habitan representan ecosistemas relativamente poco explorados, sin referencias claras en relación a los niveles de diversidad que presentan. En el presente trabajo reportamos la presencia de 216 especies y morfoespecies de invertebrados y una estimación total de riqueza entre 263 y 373 morfoespecies, para 18 charcos temporales de la cuenca de la Laguna Castillos, en el departamento de Rocha, Uruguay. Esta diversidad permitiría mantener la estabilidad del funcionamiento del ecosistema y los servicios que este brinda como ser agua potable, forraje para el ganado, limitación en la abundancia de patógenos y brotes de enfermedades o resistencia del sistema frente a contaminantes. Los estudios ecológicos en charcos temporales constituyen excelentes modelos para estudios de biodiversidad ya que proveen resultados generalizables a otros ecosistemas. Sin embargo, una de las limitaciones que encontramos en este trabajo fue la dificultad para la identificación taxonómica de los invertebrados. La gestión y manejo de la diversidad necesita una base taxonómica sólida que permita conocer la composición y estructura de dicha diversidad. Por lo tanto, resaltamos la importancia de realizar estudios en sistemática de invertebrados de agua dulce en Uruguay.

Palabras clave: Bañados de Rocha, metacomunidades, dominancia, servicios ecosistémicos.

\begin{abstract}
The hidden diversity: invertebrates of temporary ponds in Barra Grande Uruguay. Temporary ponds of Uruguay and the invertebrates that inhabit them represent relatively unexplored ecosystems, without clear references in relation to the diversity levels they host. In the present work we report the presence of 216 species and morphospecies of invertebrates and a total estimate of richness between 263 and 373 species, for 18 temporary ponds of Castillos Lagoon Basin, in the department of Rocha, Uruguay. This high species diversity would allow maintaining the stability of ecosystem functioning and the services it provides, such as drinking water, attenuation of hydrological pulses in lagoons and rivers, forage for livestock, limitation in the abundance of pathogens and disease outbreaks or system resistance against contaminants. Ecological studies in temporary ponds are excellent models for biodiversity studies and provide results that can be generalized to other ecosystems. However, one of the limitations raised in this work was the difficulty for the taxonomic identification of invertebrates. The management of diversity needs a solid taxonomic base that allows knowing the composition and structure of that diversity. Therefore, we highlight the importance of systematic studies of freshwater invertebrates in Uruguay.
\end{abstract}

Keywords: Rocha wetlands, metacommunities, dominance, ecosystem services 


\section{INTRODUCCIÓN}

La intensificación de las actividades humanas ha generado cambios en el uso del suelo, tendencias climáticas a escala global, fragmentación de hábitats e invasiones biológicas a lo largo de todo el planeta (Clemente y Brugnoli, 2002; Borthagaray, Clemente, Boccardi, Brugnoli y Muniz, 2006; Lockwood, Hoopes y Marchetti, 2007; Haddad et al., 2015). Estos fenómenos constituyen importantes amenazas para la diversidad biológica (Brugnoli, Clemente, Boccardi, Borthagaray, y Scarabino, 2005; Haddad et al., 2015). Consecuentemente, tanto desde la perspectiva teórica como la de manejo se ha puesto el foco en el desarrollo de estrategias para la conservación de la biodiversidad y el funcionamiento ecosistémico, especialmente en sistemas de agua dulce (Strayer, 2006; Collier, Probert y Jeffries, 2016; UN Environment Programme World Conservation Monitoring Centre (UNEP-WCMC), 2016; van Rees et al., 2021). No obstante, para muchos grupos taxonómicos, como los invertebrados, esto representa un gran desafío. Por un lado, se estima que solo un pequeño porcentaje de especies de insectos han sido descritos por la ciencia (Gaston, 1991; Mora, Tittensor, Adl, Simpson y Worm, 2011; Rice et al., 2018; Stork, 2018), lo cual conlleva una pérdida silenciosa de diversidad (Régnier et al., 2015). Por otro lado, este grupo ha sido postergado a razón del énfasis en otros grupos más conspicuos como mamíferos y aves, o en invertebrados carismáticos como los lepidópteros (Strayer, 2006; Balian et al., 2008). Uruguay no ha sido la excepción; de hecho, el listado de especies prioritarias para la conservación abarca solo especies de vertebrados con excepción de ciertos moluscos (Soutullo, Clavijo y MartínezLanfranco, 2013). Esto conlleva a que las priorizaciones espaciales y/o de acciones en Uruguay no tengan directamente en cuenta a los invertebrados.

La importancia de los charcos temporales ha sido reconocida por albergar una alta biodiversidad, muchas veces poco estudiada (European Pond Conservation Network (EPCN), 2008; Hill et al., 2018). Estos charcos temporales son cuerpos de agua dulce efímeros que suelen presentar un particular hidroperiodo, alternando estados activos (típicamente en las estaciones lluviosas) y secos hacia los meses más cálidos. Se encuentran ampliamente distribuidos a nivel global conformando generalmente redes de charcos (Cunillera-Montcusí et al., 2021). En su conjunto estos contienen una amplia heterogeneidad de hábitats que a menudo albergan una mayor diversidad que los cuerpos de agua dulce más grandes y permanentes, siendo claves para la dispersión y ciclo de vidas de muchas especies (Williams, Biggs, Fox, Nicolet y Whitfield, 2001). Los charcos temporales son importantes refugios tanto en paisajes urbanos como agrícolas (Chester y Robson, 2013; Davies et al., 2008), proporcionando un hábitat esencial para especies raras y amenazadas a nivel nacional e internacional. En este sentido, en charcos temporales y sus márgenes semi-acuáticos se han reportado especies incluidas en la Lista Roja de Especies Amenazadas de la UICN (EPCN, 2008).

Los invertebrados representan uno de los grupos más diversos en los sistemas acuáticos continentales, particularmente en América Latina (Ramírez y Gutiérrez-Fonseca, 2014; Vinson y Hawkins, 2003). Su importancia en los sistemas acuáticos está vinculada a su participación en distintos procesos a nivel ecosistémico, como el ciclado de nutrientes y descomposición de materia orgánica (Wallace y Webster, 1996; Graça, 2001; Collier et al., 2016). Asimismo, son fuente de recursos para otras especies y potenciales controladores de vectores biológicos o de especies con potencial de generar plagas sanitarias o productivas (Wallace y Webster, 1996). Su sensibilidad a factores de estrés ambiental también ha fundamentado su uso como indicadores del estado de los ecosistemas (Wallace y Webster, 1996; Bonada, Prat, Resh y Statzner, 2006; Chang, Lawrence, RiosTouma y Resh, 2014; Melo, Stenert, Dalzochio y Maltchik, 2015; Burwood et al., 2021). Los invertebrados acuáticos conforman un grupo taxonómico y funcionalmente diverso (Sarremejane et al., 2020), lo cual determina que no solo sus poblaciones responden a cambios en las condiciones ambientales, sino también el patrón de ensamblaje de toda la comunidad (Epele y Miserendino, 2016; Cunillera-Montcusí et al., 2020).

Uruguay presenta una importante diversidad de invertebrados de agua dulce (Bentancourt, Morelli y Scatoni, 2009), la cual ha sido relativamente poco estudiada (Gimenez, Borthagaray, Rodríguez, Brazeiro y Dimitriadis, 2005; Morelli y Verdi, 2014; Bao et al., 2021), en particular cuando se consideran los patrones de ensamblaje de comunidades y los mecanismos asociados (ej. Meerhoff et al., 2007; Borthagaray, Berazategui y Arim, 2015a; Iglesias et al., 2017; Clemente et al., 2019; Rodríguez-Tricot y Arim, 2020). Para la mayoría de las regiones del país se carece de líneas de base de diversidad (Saizar et al., 2010). Este escenario dificulta la priorización de áreas para la conservación y la detección de tendencias a gran escala en la dinámica de la biodiversidad. En el presente trabajo se releva la diversidad de invertebrados en una metacomunidad de charcos temporales del Departamento de Rocha. El estudio enfatizó un diseño de muestreo que cubriera los gradientes ambientales que potencialmente determinan la composición de invertebrados observados-área, productividad, heterogeneidad ambiental y aislamiento relativo de los charcos. Asimismo, se trabajó al máximo nivel taxonómico posible, intentando aportar una descripción confiable de la diversidad de invertebrados en el sistema de estudio y a la fecha de muestreo. 


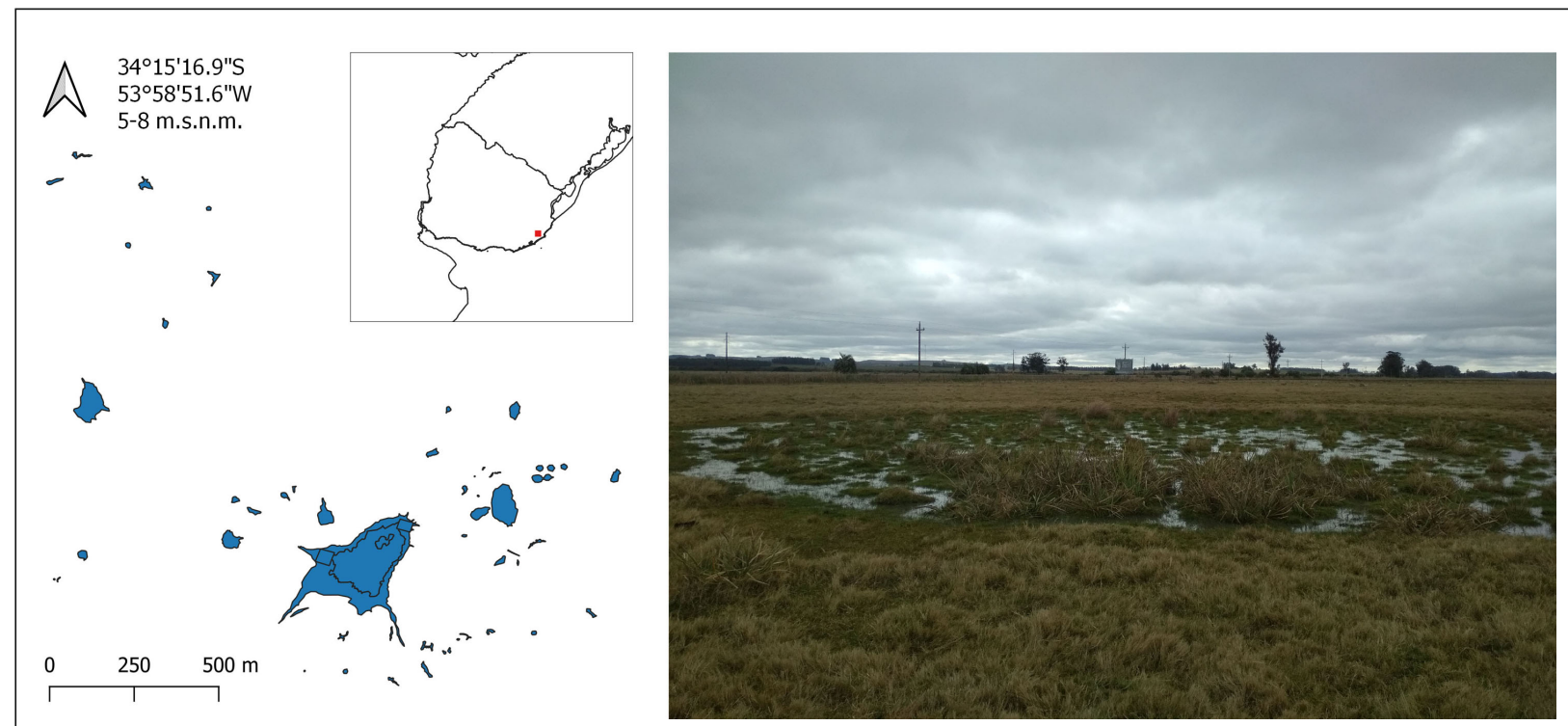

Fig. 1. Sitio de estudio. Localización y disposición espacial del sistema de charcos temporales estudiados en el Departamento de Rocha, Uruguay (izquierda). Fotografía de uno de los charcos muestreados (derecha).

\section{MATERIAL Y MÉTODOS}

\section{Área de estudio}

El área de estudio está ubicada en la cuenca de la Laguna Castillos, en el departamento de Rocha, Uruguay $\left(34^{\circ} 15^{\prime} 16.9^{\prime \prime} \mathrm{S} 53^{\circ} 58^{\prime} 51.6 " \mathrm{~W}\right.$; 5-8 metros s.n.m). Forma parte de la Reserva de la Biósfera Bañados del Este y Franja Costera (Man and the Biosphere Programme (MAB-UNESCO), Pezzani, 2007) y fue recientemente incorporada al Sistema Nacional de Áreas Protegidas (Uruguay, Decreto No. 59/020).

El sistema de estudio está compuesto por 61 charcos temporales que se forman en una matriz de pastizal inundable, en dos establecimientos privados ("Barra Grande" y "El Guri") donde se practica ganadería extensiva (Laufer et al., 2009; Arim, Abades, Laufer, Loureiro y Marquet, 2010; Arim et al. 2011; Piñeiro-Guerra, Fagúndez-Pachón, Oesterheld y Arim, 2014; Fig. 1). Los charcos están activos durante la estación lluviosa, típicamente en otoño e invierno y se secan en verano, cuando la pérdida de agua por evaporación excede a las precipitaciones. En conjunto abarcan un amplio rango de áreas (cinco órdenes de magnitud), condiciones locales como forma, profundidad, y materia orgánica (Laufer et al., 2009), y aislamiento relativo (Borthagaray et al., 2015a; Borthagaray, Pinelli, Berazategui, Rodríguez-Tricot y Arim, 2015b). Presentan un gradiente en heterogeneidad ambiental asociado a la densidad de montículos de tierra que sobresalen de la superficie de agua, que afectan los niveles de productividad y diversidad (Piñeiro-Guerra et al., 2014; Pinelli, 2016, Rodríguez-Tricot y Arim 2020). Existe una alta diversidad de peces, anfibios, invertebrados y plantas en la metacomunidad, generando un gradiente de riqueza en los principales grupos biológicos que habitan estos charcos (Arim et al., 2011; PiñeiroGuerra et al., 2014; Canavero, Hernández, Zarucki, y Arim, 2014; Borthagaray et al., 2015a).

\section{Muestreo}

Los relevamientos de diversidad de invertebrados fueron realizados en octubre de 2008, dónde sólo 18 de los charcos se encontraban activos, i.e.: contenían agua. La forma de los charcos se aproximó a la de una elipse. Se establecieron las unidades de muestreo a lo largo del eje mayor y menor según la longitud de los mismos, equiparando de esta forma el esfuerzo de muestreo en charcos de distinto tamaño (Arim et al., 2011). Cuando la longitud de los ejes midió entre 10 y $50 \mathrm{~m}$ se definieron 5 unidades de muestreo equidistantes. En los ejes menores a 10 y mayores a $50 \mathrm{~m}$, se consideró una unidad de muestreo cada dos y diez metros, respectivamente. Las muestras fueron tomadas con calderines de $15 \times 20 \mathrm{~cm}$ y un milimetro de malla. En cada unidad de muestreo se pasó el calderín de forma estandarizada: tres veces horizontalmente abarcando una superficie de aproximadamente un $\mathrm{m}^{2}$, y tres veces verticalmente, desde el fondo del charco hasta la superficie (Arim et al., 2011; Canavero et al., 2014).

El procesamiento primario de las muestras (lavado y separación gruesa) se realizó en laboratorio en los 


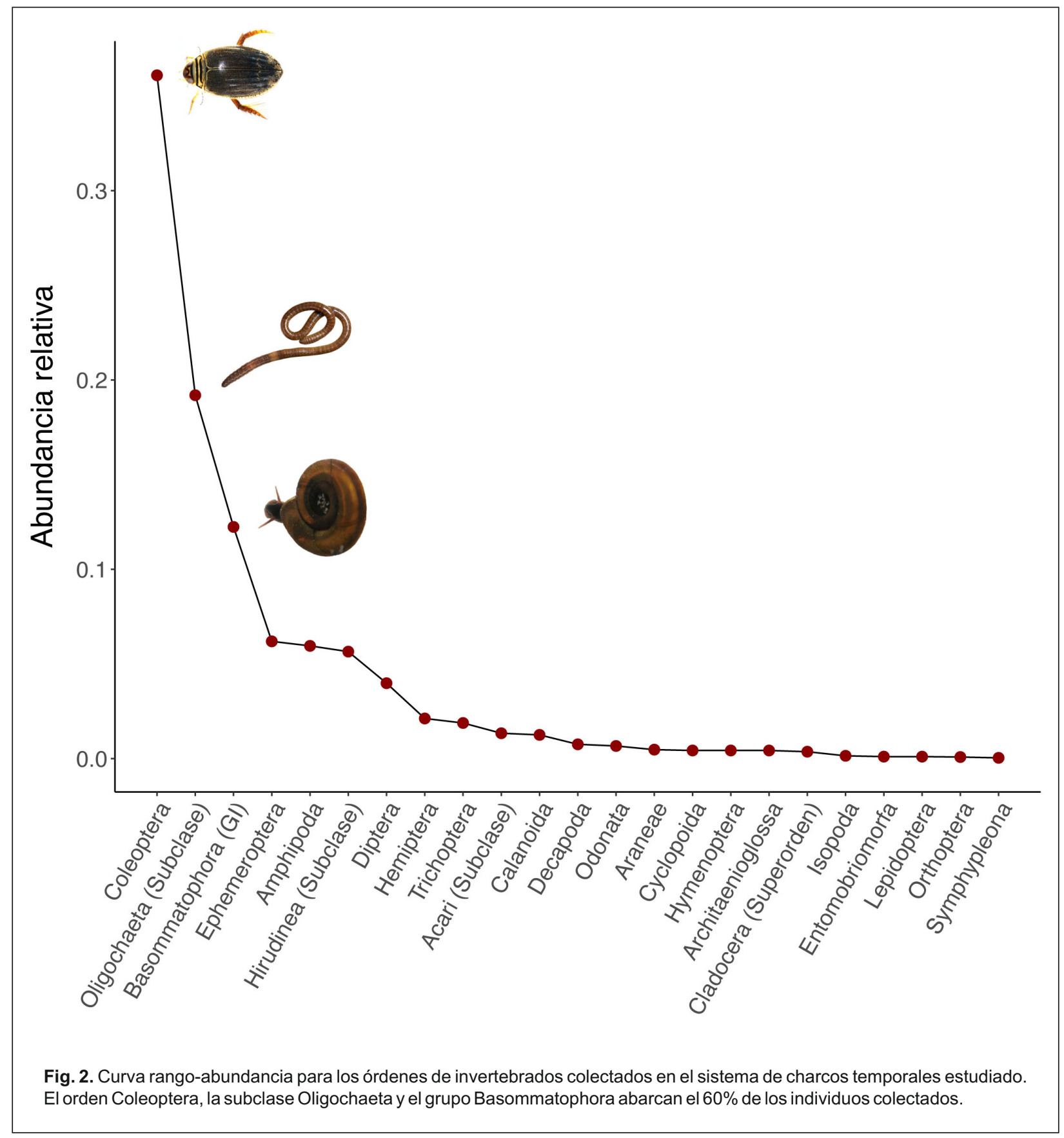

días inmediatos al muestreo. Las muestras fueron conservadas en alcohol al 95\% para su identificación. Los individuos fueron identificados con el mayor grado de precisión taxonómica posible, con la ayuda de claves de identificación y bibliografía de referencia (Peña, 1996; Marvaldi y Lanteri, 2005; Heckmann, 2006; 2008; Bentancourt et al., 2009; Dominguez y Fernández, 2009). En los casos que no fue posible alcanzar una resolución taxonómica mayor, los individuos fueron asignados a taxones fácilmente separables por diferencias morfológicas (i.e. morfoespecies o la categoría de menor jerarquía posible), asociados a una colección de referencia.

\section{Análisis de datos}

La descripción de las abundancias relativas se realizó por medio de la construcción de curvas rangoabundancia para dos niveles taxonómicos (orden y especie/ morfoespecie). Considerando las dificultades prácticas que implica alcanzar censos completos (Gotelli y Colwell, 2011), es esperable que haya un subregistro del total de especies que efectivamente 
existen en el sistema. Para dar cuenta de este subregistro se estimó el número de especies esperado (más allá del observado en el muestreo), a partir del índice de riqueza de Chao 1 (con un intervalo de confianza del 95\%) (Chao, 1987; Chao y Chiu, 2016). Este índice estima la riqueza total esperada dando cuenta de la heterogeneidad en las abundancias de los diferentes grupos y considerando explícitamente el número de especies con abundancia de uno y dos individuos. La estimación de la riqueza esperada se realizó usando el paquete iNEXT (Hsieh, Ma y Chao, 2016) del software estadístico R (R Core Team, 2021).

\section{RESULTADOS}

Se registraron 216 especies/ morfoespecies de invertebrados acuáticos y terrestres en distintos estadíos de vida, en un total de 5056 individuos colectados. De las 216 especies/morfoespecies sólo siete corresponden a una resolución de identificación a nivel de especies, las 209 restantes fueron identificadas a distintas categorías taxonómicas de mayor jerarquía. En total, fue posible identificar a partir de la muestra un total de cinco Phyla, 13 Clases, 23 Ordenes (incluyendo el Grupo Informal Basommatophora y 3 subclases: Acari, Hirudinea y Oligochaeta), 72 Familias y 58 géneros diferentes (Tabla 1 y Material Suplementario, Tabla S1).

Las curvas de rango-abundancia muestran que los grupos más abundantes corresponden al orden Coleóptera, la subclase Oligochaeta y el grupo Basommatophora (Fig. 2). Estos tres grupos comprenden más del $60 \%$ de los individuos colectados. A su vez, las morfoespecies más abundantes pertenecen a los grupos Oligochaeta, Coleoptera, Ostracoda y Basommatophora constituyendo el $35 \%$ de la abundancia total (Fig. 3). Adicionalmente, del total de especies y morfoespecies identificadas 159 presentan una abundancia menor o igual a 10 individuos, y 18, 31 y 76 de estas especies mostraron una abundancia igual a tres, dos y un individuo respectivamente. El número de especies esperado del sistema, basado en el índice de riqueza de Chao 1 y calculado en función de las especies/ morfoespecies con abundancia igual a uno y dos individuos, fue de 302 (IC 95\%: 263-373), suponiendo una diferencia de 86 especies respecto a las observadas en el muestreo.

\section{DISCUSIÓN}

Los estudios sobre los patrones y procesos relativos a la biodiversidad presentan un sesgo crónico hacia grupos biológicos conspicuos y Uruguay no es una excepción en este sentido (Strayer, 2006; Morelli y Verdi, 2014; Collier et al., 2016). En un muestreo puntual de un área relativamente chica del Departamento de Rocha reportamos 216 especies/ morfoespecies de invertebrados y una estimación total de riqueza entre 263 y 373 especies. Esta información provee una referencia para la región y el tipo de ecosistema en relación a los niveles de biodiversidad esperados. Es preciso mencionar que este reporte incluye también invertebrados no necesariamente acuáticos (ej. hormigas, arañas). Estos se encuentran típicamente asociados a los límites y los montículos terrestres presentes en los charcos, y por lo tanto fueron capturados en el muestreo. A pesar de haber consultado la bibliografía de referencia, no fue posible alcanzar el nivel de especie en la mayoría de los taxa analizados. Esto resalta la importancia de realizar estudios en sistemática de invertebrados de agua dulce en Uruguay, y de dedicar recursos en este sentido (Giangrande, 2003). Se trata de un área en donde hay un interesante terreno para avanzar en la identificación de especies, sus relaciones evolutivas y los contextos ecológicos en que ocurrieron (ej. Loureiro et al., 2015; 2018).

La taxonomía o el conjunto de herramientas para delimitar especies muchas veces tiende a quedar relegada en la academia, si bien contar con taxónomos expertos y bien entrenados es muchas veces un prerrequisito para generar una base de prioridades de conservación, empezando por los listados de especies (Johnson et al., 2018; Mace, 2004). En este sentido, la conservación y la taxonomía están estrechamente ligadas, dado que la gestión y manejo de la diversidad necesita una base taxonómica sólida que permita conocer la composición y estructura de dicha diversidad (Mace, 2004). Esto es particularmente importante en grupos megadiversos, como es el caso de los invertebrados, que muchas veces son excluidos de los listados y políticas de priorización de la conservación debido a la incapacidad de basarse en datos e información robusta y de calidad (Hochkirch et al., 2020).

La diversidad de invertebrados identificada en el área de estudio puede relacionarse con múltiples servicios ecosistémicos. En primer lugar, en los charcos muestreados se han identificado tanto potenciales parásitos y vectores biológicos, como hirudíneos, gasterópodos y culícidos (Ponce de León, 1989; Volonterio, López-de León y de León, 2004; Volonterio y Ponce de León, 2005; Mas-Comas, Bargues y Valero, 2005; Neill y Arim, 2011; Araujo, Santos, Peçanha, y Machado da Silva, 2013), pero también, la presencia de especies con el potencial de limitar el efecto de estos vectores en la diseminación de enfermedades. Por ejemplo, la depredación por peces anuales (Laufer et al., 2009) y la competencia con otras especies del gremio (Rodríguez-Tricot, 2016) puede limitar la distribución y abundancia de estos vectores, manteniéndolos por debajo de los valores necesarios para la generación de problemas 


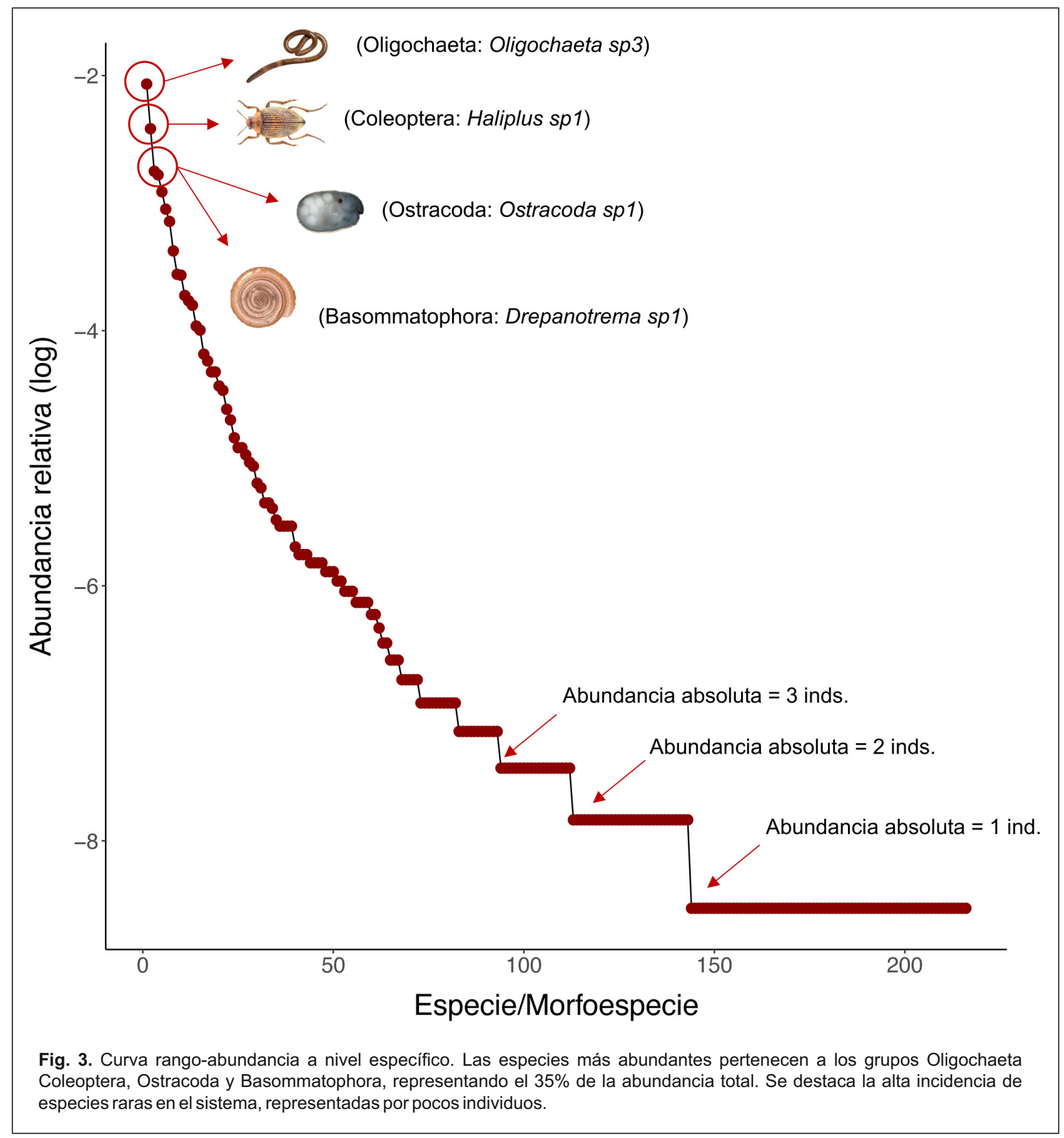

sanitarios. En particular, los ditíscidos (Orden: Coleoptera), uno de los grupos más abundantes de este sistema, son depredadores de mosquitos llegando a tener un rol importante en su control, y en general en la red trófica de sistemas acuáticos (Wallace y Webster, 1996; Jäch y Balke, 2008). En segundo lugar, los altos niveles de diversidad implicarían una mayor resistencia a invasiones biológicas y sus problemáticas asociadas (Clemente y Brugnoli, 2002; Muniz, Clemente y Brugnoli, 2005; Fargione y Tilman, 2005; Lockwood et al., 2007). En tercer lugar, destacamos el potencial de sistemas diversos con redes tróficas complejas para atenuar el efecto de los contaminantes ambientales en el funcionamiento de los sistemas (Garay-Narváez, Arim, Flores y Ramos-Jiliberto, 2013), el intercambio de carbono con la atmósfera (Schindler, Carpenter, Cole, Kitchell y Pace, 1997) o la limitación de blooms de algas y eutrofización de los cuerpos de agua (Chapin et al., 2000). Asimismo, la alta diversidad tiene el potencial de atenuar las variaciones a nivel ecosistémico manteniendo estable los servicios que 
Tabla 1. Listado de órdenes de invertebrados que conforman la metacomunidad de charcos temporales en el Departamento de Rocha, Uruguay. Se indican los taxa que no fue posible identificar a nivel de orden (GI: Grupo Informal, Subclase, Clase, Filo).

\begin{tabular}{|c|c|c|c|c|}
\hline Filo & Clase & Orden & Riqueza & Abundancia \\
\hline \multirow[t]{2}{*}{ Annelida } & Clitellata & Oligochaeta (Subclase) & 6 & 886 \\
\hline & & Hirudinea (Subclase) & 9 & 261 \\
\hline \multirow[t]{20}{*}{ Arthropoda } & Arachnida & Acari (Subclase) & 9 & 62 \\
\hline & & Araneae & 15 & 22 \\
\hline & Branchiopoda & Anomopoda & 3 & 17 \\
\hline & Collembola & Entomobriomorfa & 2 & 5 \\
\hline & & Symphypleona & 1 & 2 \\
\hline & Hexanauplia & Cyclopoida & 1 & 20 \\
\hline & Insecta & Coleoptera & 66 & 1666 \\
\hline & & Diptera & 37 & 184 \\
\hline & & Ephemeroptera & 5 & 286 \\
\hline & & Hemiptera & 20 & 98 \\
\hline & & Hymenoptera & 9 & 20 \\
\hline & & Lepidoptera & 3 & 5 \\
\hline & & Odonata & 4 & 31 \\
\hline & & Orthoptera & 4 & 4 \\
\hline & & Trichoptera & 4 & 87 \\
\hline & Malacostraca & Amphipoda & 1 & 275 \\
\hline & & Decapoda & 1 & 35 \\
\hline & & Isopoda & 2 & 7 \\
\hline & Maxillopoda & Calanoida & 1 & 58 \\
\hline & & Ostracoda & 23 & 24 \\
\hline Cnidaria & Hydrozoa & & 1 & 8 \\
\hline \multirow[t]{3}{*}{ Mollusca } & Bivalvia & & 2 & 15 \\
\hline & Gasteropoda & Architaenioglossa & 1 & 20 \\
\hline & & Basommatophora (GI) & 6 & 565 \\
\hline Nematoda & & & 1 & 93 \\
\hline Total & & & 216 & 5056 \\
\hline
\end{tabular}

este provee (Tilman, Isbell y Cowles, 2014). La simplificación del sistema por pérdida de charcos y reducción de sus áreas, puede brindar el contexto para la dominancia de pocas especies, favoreciendo dinámicas ecosistémicas más erráticas con blooms de productores primarios, irrupción de enfermedades, pérdida de calidad de aguas y de la vegetación del sistema (Mori, Isbell y Seidl, 2018; de Meester et al., 2005).

La alta diversidad asociada a los charcos temporales, y los servicios ecosistémicos con los que se relaciona, sustentan la necesidad y ventajas de preservar los ecosistemas de charcos temporales. Es posible que el carácter temporal de estos sistemas los haga pasar desapercibidos a los ojos de los gestores/tomadores de decisiones y el público general. Sin embargo, entre otras características que los hacen únicos, los charcos temporales dan soporte a una biota particular, adaptada a la temporalidad de estos sistemas. Teniendo en cuenta que pueden ser una fracción pequeña del paisaje, su contribución a la diversidad muchas veces es desproporcionadamente grande (Boix, Sala, Gascón, Ruhí y Quintana, 2009). Lamentablemente, los charcos temporales no son generalmente identificados como sistemas relevantes para la conservación (Collinson et al., 1995; Boix et al., 2009). De hecho, el secado de humedales y charcos es una práctica observada en la región de estudio. En buena medida, esto se debe a la ausencia de una comprensión fundada sobre los servicios ecosistémicos brindados por estas comunidades y su relevancia en la preservación de la biodiversidad. La cuantificación científica de estos servicios y su difusión a los tomadores de decisiones y propietarios de campos es fundamental para la preservación de estos sistemas a largo plazo.

Por último, cabe resaltar el papel de los charcos temporales como modelos de estudio en ecología. Se 
trata de comunidades complejas, diversas y ensambladas en gradientes ambientales relevantes por su efecto en los mecanismos ecológicos de interés (Arim et al. 2010, 2011). En el sistema de estudio considerado aquí, los charcos presentan casi seis órdenes de magnitud en sus áreas (Arim et al., 2011), pero también importantes gradientes en sus aislamientos espaciales (Borthagaray et al., 2015a; 2015b), heterogeneidad interna (Rodríguez-Tricot y Arim, 2020) o biomasa de productores primarios (Piñeiro-Guerra et al., 2014). Asociados a estos gradientes las comunidades presentan diferentes estructuras tróficas, diversidades y patrones de ensamblaje comunitario (Piñeiro-Guerra et al., 2014; Canavero et al., 2014; Borthagaray et al., 2015b; Meerhoff et al., 2007; Rodríguez-Tricot y Arim, 2020). El muestreo de un charco temporal típicamente requiere menos de dos horas de trabajo en campo, permitiendo relevar decenas de comunidades en una sola campaña de muestreo. Por todo esto, el sistema de estudio en particular, y los charcos temporales en general, cumplen con las condiciones de ser manejables desde una perspectiva logística, ser realistas y proveer resultados generalizables a otros sistemas de estudio (Sirvastaba et al., 2004). Consecuentemente, estos microcosmos naturales representan un modelo de estudio excepcional en ecología (Sirvastaba et al., 2004). Un foco más generalizado en la evaluación y construcción de teoría ecológica tiene mucho para ganar en la identificación y análisis de modelos de estudio realistas como han mostrado ser los charcos temporales.

\section{AGRADECIMIENTOS}

Los autores del trabajo deseamos agradecer la persistente influencia del Dr. Juan María Clemente ("Checho") en nuestro interés y comprensión de la diversidad de invertebrados de agua dulce y su importancia en el funcionamiento de los ecosistemas y los servicios que estos brindan. Su fascinación por estos organismos y la identificación de problemáticas ambientales asociadas como las bioinvasiones, han sembrado un duradero impacto en nuestra comunidad académica, del cual estamos sinceramente agradecidos.

Agradecemos también a los establecimientos "Barra Grande" y "El Guri" por brindarnos facilidades para el trabajo de campo durante 18 años. Los autores agradecen al proyecto CSIC-Grupos-657725.

\section{BIBLIOGRAFÍA}

Araujo, J.C., Santos, B., Peçanha, R., y Machado da Silva, A.V. (2013). Haementeria lutzi Pinto, 1920 (Hirudinea: Glossiphoniidae) as a putative Vector of Trypanosoma evansi (Kinetoplastida: Trypanosomatidae) in the Pantanal
Matogrossense (MS, Brazil). Entomology, Ornithology \& Herpetology, 2(2), 108.

Arim, M., Abades, S.R., Laufer, G., Loureiro, M., y Marquet, P.A. (2010). Food web structure and body size: trophic position and resource acquisition. Oikos, 119(1), 147-153.

Arim, M., Berazategui, M., Barreneche, J.M., Ziegler, L., Zarucki, M., y Abades, S.R. (2011). Determinants of density-body size scaling within food webs and tools for their detection. Advances in Ecological Research, 45, 1-39.

Balian, E.V., Segers, H., Lévèque, C. y Martens, K. (2008). The Freshwater Animal Diversity Assessment: an overview of the results. Hydrobiologia, 595, 627-637.

Bao, L., Martínez, S., Cadenazzi, M., Urrutia, M., Seijas, L., y Castiglioni, E. (2021). Aquatic macroinvertebrates in Uruguayan rice agroecosystem. Biodiversity Data Journal, 9.

Bentancourt Pérez, C.M., Morelli Mazzeo, E.R., y Scatoni, I.B. (2009). Insectos del Uruguay, 595(899), 589-623

Boix, D., J. Sala, S. Gascón, A. Ruhí, y X. Quintana. (2009). Structure of invertebrate assemblages: contribution to the ecological functioning of the Mediterranean temporary ponds. International conference on mediterranean temporary ponds: proceedings and Abstracts Maó,151-187.

Bonada, N., Prat, N., Resh, V.H., y Statzner, B. (2006). Developments in aquatic insect biomonitoring: a comparative analysis of recent approaches. Annual Review of Entomology, 51, 495-523.

Borthagaray, A.I., Clemente, J.M., Boccardi, L., Brugnoli, E., y Muniz, P. (2006). Impacto potencial de invasión de Ficopomatus enigmaticus (Fauvel) (Polychaeta: Serpulidae) en la Laguna de Rocha, Uruguay. PanAmerican Journal of Aquatic Sciences, 1(1), 57-65.

Borthagaray, A.I., Berazategui, M., y Arim, M. (2015a). Disentangling the effects of local and regional processes on biodiversity patterns through taxon-contingent metacommunity network analysis. Oikos, 124(10), 1383-1390.

Borthagaray, A.I., Pinelli, V., Berazategui, M., Rodríguez-Tricot, L., y Arim, M. (2015b). Effects of metacommunity networks on local community structures: from theoretical predictions to empirical evaluations. En: A.Belgrano, G. Woodward y U. Jacob (Eds.). Aquatic functional biodiversity (pp. 75-111). Academic Press.

Brugnoli, E., Clemente, J., Boccardi, L., Borthagaray, A., y Scarabino, F. (2005). Golden mussel Limnoperna fortunei (Bivalvia: Mytilidae) distribution in the main hydrographical basins of Uruguay: update and predictions. Anais da Academia Brasileira de Ciências, 77(2), 235-244.

Burwood, M., Clemente, J., Meerhoff, M., Iglesias, C., 
Goyenola, G., Fosalba, C., ... Teixeira de Mello, F. (2021). Macroinvertebrate communities and macrophyte decomposition could be affected by land use intensification in subtropical lowland streams. Limnetica, 40(2), 343-357.

Canavero, A., Hernández, D., Zarucki, M., y Arim, M. (2014). Patterns of co-occurrences in a killifish metacommunity are more related with body size than with species identity. Austral Ecology, 39(4), 455-461.

Chang, F.H., Lawrence, J.E., Rios-Touma, B., y Resh, V.H. (2014). Tolerance values of benthic macroinvertebrates for stream biomonitoring: assessment of assumptions underlying scoring systems worldwide. Environmental monitoring and assessment, 186(4), 2135-2149.

Chao, A. (1987). Estimating the population size for capture-recapture data with unequal catchability. Biometrics, 783-791.

Chao, A., y Chiu, C.H. (2016). Nonparametric estimation and comparison of species richness. eLS, 1-11.

Chapin lii, F.S., Zavaleta, E.S., Eviner, V.T., Naylor, R.L., Vitousek, P.M., Reynolds, H.L., ... Díaz, S. (2000). Consequences of changing biodiversity. Nature, 405(6783), 234-242.

Chester, E.T., y Robson, B.J. (2013). Anthropogenic refuges for freshwater biodiversity: their ecological characteristics and management. Biological Conservation, 166, 64-75.

Clemente, J.M., Boll, T., Teixeira-de Mello, F., Iglesias, C., Roer, A., Pedersen, E.J., y Meerhoff, M. (2019). Role of plant architecture on littoral macroinvertebrates in temperate and subtropical shallow lakes: a comparative manipulative field experiment. Limnetica, 38(2), 759-772.

Clemente, J.M., y Brugnoli, E. (2002). First record of Limnoperna fortunei (Dunker, 1857) (Bivalvia: Mytilldae) in continental waters of Uruguay (Rio Negro and Rio Yi). Boletín de la Sociedad Zoológica del Uruguay, 13, 29-33.

Collier, K.J., Probert, P.K., y Jeffries, M. (2016). Conservation of aquatic invertebrates: concerns, challenges and conundrums. Aquatic Conservation: Marine and Freshwater Ecosystems, 26(5), 817-837.

Collinson, N.H., Biggs, J., Corfield, A.H. M.J., Hodson, M.J., Walker, D., Whitfield, M., y Williams, P.J. (1995). Temporary and permanent ponds: an assessment of the effects of drying out on the conservation value of aquatic macroinvertebrate communities. Biological conservation, 74(2), 125-133.

Cunillera-Montcusí, D., Borthagaray, A.I., Boix, D., Gascón, S., Sala, J., Tornero, I., ... Arim, M. (2021). Metacommunity resilience against simulated gradients of wildfire: disturbance intensity and species dispersal ability determine landscape recover capacity. Ecography, 44(7).

Cunillera-Montcusí, D., Arim, M., Gascón, S., Tornero, I., Sala, J., Boix, D., y Borthagaray, A.I. (2020). Addressing trait selection patterns in temporary ponds in response to wildfire disturbance and seasonal succession. Journal of Animal Ecology, 89(9), 2134-2144.

De Meester, L., Declerck, S., Stoks, R., Louette, G., Van De Meutter, F., De Bie, T., ... \& Brendonck, L. (2005). Ponds and pools as model systems in conservation biology, ecology and evolutionary biology. Aquatic conservation: Marine and freshwater ecosystems, 15(6), 715-725.

Davies, B., Biggs, J., Williams, P., Whitfield, M., Nicolet, P., Sear, D., ... Maund, S. (2008). Comparative biodiversity of aquatic habitats in the European agricultural landscape. Agriculture, Ecosystems \& Environment, 125(1-4), 1-8.

Domínguez, E., y Fernández, H.R. (2009). Macroinvertebrados bentónicos sudamericanos. Sistemática y biología. Fundación Miguel Lillo, Tucumán, Argentina, 656.

Epele, L.B., y Miserendino, M.L. (2016). Temporal dynamics of invertebrate and aquatic plant communities at three intermittent ponds in livestock grazed Patagonian wetlands. Journal of Natural History, 50(11-12), 711-730.

EPCN (2008). The Pond Manifesto; European Pond Conservation Network. https:// freshwaterhabitats.org.uk/wpcontent/uploads/2016/06/EPCNMANIFESTO.pdf [Consulta: 10 de junio de 2021].

Fargione, J.E., y Tilman, D. (2005). Diversity decreases invasion via both sampling and complementarity effects. Ecology Letters, 8(6), 604-611.

Gaston, K.J. (1991). The magnitude of global insect species richness. Conservation biology, 5(3), 283-296.

Garay-Narváez, L., Arim, M., Flores, J.D., y Ramos-Jiliberto, R. (2013). The more polluted the environment, the more important biodiversity is for food web stability. Oikos, 122(8), 1247-1253.

Gimenez, L., Borthagaray, A.I., Rodríguez, M., Brazeiro, A., y Dimitriadis, C. (2005). Scaledependent patterns of macrofaunal distribution in soft-sediment intertidal habitats along a largescale estuarine gradient. Helgoland Marine Research, 59(3), 224-236.

Graça, M.A. (2001). The role of invertebrates on leaf litter decomposition in streams-a review. International Review of Hydrobiology: A Journal Covering all Aspects of Limnology and Marine Biology, 86(4-5), 383-393 
Giangrande, A. (2003). Biodiversity, conservation, and the 'Taxonomic impediment'. Aquatic Conservation: Marine and Freshwater Ecosystems, 13(5), 451-459.

Gotelli, N.J., y Colwell, R.K. (2011). Estimating species richness. Biological diversity: frontiers in measurement and assessment, 12(39-54), 35.

Haddad, N.M., Brudvig, L.A., Clobert, J., Davies, K.F., Gonzalez, A., Holt, R.D., ... Townshend, J.R. (2015). Habitat fragmentation and its lasting impact on Earth's ecosystems. Science advances, 1(2), e1500052.

Heckman, C.W. (2006). Encyclopedia of South American Aquatic Insects: Odonata Zygoptera. Illustrated Keys to Known Families, Genera, and Species in South America. Springer.

Heckman, C.W. (2008). Encyclopedia of South American Aquatic Insects: Odonata Anisoptera. Illustrated Keys to Known Families, Genera, and Species in South America. Springer.

Hill, M.J., Hassall, C., Oertli, B., Fahrig, L., Robson, B.J., Biggs, J., ... Wood, P.J. (2018). New policy directions for global pond conservation. Conservation Letters, 11(5), e12447.

Hochkirch, A., Samways, M.J., Gerlach, J., Böhm, M., Williams, P., Cardoso, P., ... Dijkstra, K.D.B. (2021). Astrategy for the next decade to address data deficiency in neglected biodiversity. Conservation Biology, 35(2), 502-509.

Hsieh, T.C., Ma, K.H., y Chao, A. (2016). iNEXT: an R package for rarefaction and extrapolation of species diversity ( $\mathrm{H}$ ill numbers). Methods in Ecology and Evolution, 7(12), 1451-1456.

Iglesias, C., Jeppesen, E., Mazzeo, N., Pacheco, J.P., Mello, F.T.D., Landkildehus, F., ... Meerhoff, M. (2017). Fish but not macroinvertebrates promote trophic cascading effects in high density submersed plant experimental lake food webs in two contrasting climate regions. Water, 9(7), 514.

Jäch, M.A., \& Balke, M. (2008). Global diversity of water beetles (Coleoptera) in freshwater. Freshwater animal diversity assessment, Hydrobiologia, 595(1), 419-442.

Johnson, N.A., Smith, C.H., Pfeiffer, J.M., Randklev, C.R., Williams, J.D., y Austin, J.D. (2018). Integrative taxonomy resolves taxonomic uncertainty for freshwater mussels being considered for protection under the US Endangered Species Act. Scientific reports, 8(1), 1-16.

Laufer, G., Arim, M., Loureiro, M., Piñeiro-Guerra, J.M., Clavijo-Baquet, S., y Fagúndez, C. (2009). Diet of four annual killifishes: an intra and interspecific comparison. Neotropical Ichthyology, 7(1), 77-86.
Lockwood, J.L., M.F. Hoopes, y M.P. Marchetti. 2007, Invasion Ecology. London, Wiley-Blackwell.

Loureiro, M., Borthagaray, A., Hernández, D., Duarte, A., Pinelli, V., y Arim, M. (2015). Austrolebias in space: scaling from ponds to biogeographical regions. Annual fishes: Life history strategy, diversity, and evolution. CRC Press, Boca Ratón, 111-132.

Loureiro, M., Sá, R.D., Serra, S.W., Alonso, F., Lanés, L.E.K., Volcan, M.V., ... Garcia, G. (2018). Review of the family Rivulidae (Cyprinodontiformes, Aplocheiloidei) and a molecular and morphological phylogeny of the annual fish genus Austrolebias Costa 1998. Neotropical Ichthyology, 16(3), e180007.

Mace, G.M. (2004). The role of taxonomy in species conservation. Philosophical Transactions of the Royal Society of London. Series B: Biological Sciences, 359(1444), 711-719.

Marvaldi, A.E., y Lanteri, A.A. (2005). Clave de taxones superiores de gorgojos sudamericanos basada en caracteres de los adultos (Coleoptera, Curculionoidea). Revista chilena de historia natural, 78(1), 65-87.

Mas-Coma, S., Bargues, M. D., y Valero, M. A. (2005). Fascioliasis and other plant-borne trematode zoonoses. International journal for parasitology, 35(11-12), 1255-1278.

Meerhoff, M., Clemente, J.M., Teixeira-de Mello, F., Iglesias, C., Pedersen, A.R., y Jeppesen, E. (2007). Can warm climate-related structure of littoral predator assemblies weaken the clear water state in shallow lakes?. Global Change Biology, 13(9), 1888-1897.

Melo, S., Stenert, C., Dalzochio, M.S., y Maltchik, L. (2015). Development of a multimetric index based on aquatic macroinvertebrate communities to assess water quality of rice fields in southern Brazil. Hydrobiologia, 742(1), $1-14$.

Mora, C., Tittensor, D.P., Adl, S., Simpson, A.G., y Worm, B. (2011). How many species are there on Earth and in the ocean?. PLoS Biol, 9(8), e1001127.

Morelli, E., y Verdi, A. (2014). Diversidad de macroinvertebrados acuáticos en cursos de agua dulce con vegetación ribereña nativa de Uruguay. Revista mexicana de biodiversidad, 85(4), 1160-1170.

Mori, A.S., Isbell, F., y Seidl, R. (2018). $\beta$-diversity, community assembly, and ecosystem functioning. Trends in ecology \& evolution, 33(7), 549-564.

Muniz, P., Clemente, J., y Brugnoli, E. (2005). Benthic invasive pests in Uruguay: a new problem or an old one recently perceived?. Marine Pollution Bulletin, 50(9), 1014-1018.

Neill, P.E., y Arim, M. (2011). Human health link to 
invasive species. En: J. O. Nriagu (Ed.). Encyclopedia of Environmental Health (pp. 116123). Burlington, USA: Elseiver.

Peña, L.E. (1996). Introducción al estudio de los insectos de Chile. Editorial Universitaria.

Pezzani, F. (2007). Reserva de biosfera bañados del este, Uruguay. Documentos de trabajo, 37.

Pinelli, V. (2016). El ambiente y la estructura comunitaria como determinantes de la estabilidad en comunidades vegetales de charcos temporales, (Tesis de Maestría). Montevideo, Uruguay: PEDECIBA, Universidad de la República.

Piñeiro-Guerra, J.M., Fagúndez-Pachón, C., Oesterheld, M., y Arim, M. (2014). Biodiversity-productivity relationship in ponds: Community and metacommunity patterns along time and environmental gradients. Austral Ecology, 39(7), 808-818.

Ponce de León, R. (1989). Description of Temnocephala haswelli n. sp. (Platyhelminthes) from the mantle cavity of Pomacea canaliculata (Lamarck). The Journal of parasitology, 524-526.

R Core Team (2021). R: A language and environment for statistical computing. $R$ Foundation for Statistical Computing, Vienna, Austria. URL https://www.R-project.org/.

Ramírez, A., y Gutiérrez-Fonseca, P.E. (2014). Estudios sobre macroinvertebrados acuáticos en América Latina: avances recientes y direcciones futuras. Revista de Biología Tropical, 62, 9-20.

Régnier, C., Achaz, G., Lambert, A., Cowie, R.H., Bouchet, P., y Fontaine, B. (2015). Mass extinction in poorly known taxa. Proceedings of the National Academy of Sciences, 112(25), 7761-7766.

Rice, J., Seixas, C.S., Zaccagnini, M.E., BedoyaGaitán, M., Valderrama, N., Anderson, C.B., ... Farinaci, J.S. (2018). Summary for policymakers of the regional assessment report on biodiversity and ecosystem services for the Americas of the Intergovernmental SciencePolicy Platform on Biodiversity and Ecosystem Services. IPBES book.

Rodríguez-Tricot, L. (2016). Estructura del paisaje y coexistencia de especies en una metacomunidad de charcos temporales, (Tesis de Maestría). Montevideo, Uruguay: PEDECIBA, Universidad de la República.

Rodríguez-Tricot, L., y Arim, M. (2020). From Hutchinsonian ratios to spatial scaling theory: the interplay among limiting similarity, body size and landscape structure. Ecography, 43(2), 318-327.

Saizar, C., Boccardi, L., Clemente, J., Dabezies, M.,
Ferrari, G., Caramés, D.M., ... Tana, J. (2010). Línea de base para evaluar el impacto de una planta de celulosa en el Río Uruguay. Innotec, 5, 11-22.

Sarremejane, R., Puey, N.C., Datry, T., Stubbington, R., Alp, M., Canedo-Arguelles, M., ... Bonada, N. (2020). DISPERSE: A trait database to assess the dispersal potential of aquatic macroinvertebrates. Scientific data, 7(1), 1-9.

Schindler, D.E., Carpenter, S.R., Cole, J.J., Kitchell, J.F., y Pace, M.L. (1997). Influence of food web structure on carbon exchange between lakes and the atmosphere. Science, 277(5323), 248-251.

Soutullo, A., Clavijo, C., y Martínez-Lanfranco, J.A. (2013). Especies prioritarias para la conservación en Uruguay. Vertebrados, moluscos continentales y plantas vasculares. M o n t e v i d e o, U r u g u a y : SNAP/DINAMA/MVOTMA Y DICYT/MEC

Srivastava, D.S., Kolasa, J., Bengtsson, J., Gonzalez, A., Lawler, S.P., Miller, T.E., ... Trzcinski, M.K. (2004). Are natural microcosms useful model systems for ecology?. Trends in ecology \& evolution, 19(7), 379-384.

Stork, N.E. (2018). How many species of insects and other terrestrial arthropods are there on Earth?. Annual review of entomology, 63, 31-45.

Strayer, D.L. (2006). Challenges for freshwater invertebrate conservation. Journal of the North American Benthological Society, 25(2), 271-287.

Tilman, D., Isbell, F., y Cowles, J.M. (2014). Biodiversity and ecosystem functioning. Annual review of ecology, evolution, and systematics, 45, 471-493.

UN Environment Programme World Conservation Monitoring Centre. (2016). El estado de la biodiversidad en América Latina y el Caribe. Cambridge, Reino Unido: UNEP-WCMC.

Uruguay. Decreto No. 59/020. Delimitación y clasificación del Área Natural Protegida "Laguna de Castillos". Recuperado de: https://www.impo.com.uy/bases/decretos/592020/1 [Consulta: 7 de junio de 2021].

van Rees, C.B., Waylen, K.A., Schmidt-Kloiber, A., Thackeray, S.J., Kalinkat, G., Martens, K., ... Jähnig, S.C. (2021). Safeguarding freshwater life beyond 2020: Recommendations for the new global biodiversity framework from the European experience. Conservation Letters, 14(1), e12771.

Vinson, M.R., y Hawkins, C.P. (2003). Broad-scale geographical patterns in local stream insect genera richness. Ecography, 26(6), 751-767.

Volonterio, O., López-de León, E., y de León, R.P. (2004). Infestation dynamics and 
histopathology of two species of freshwater leeches (Hirudinea: Piscicolidae) on teleost fish from Uruguay. Comparative parasitology, 71(1), $21-28$.

Volonterio, O., y Ponce de León, R. (2005). Description of Dendrorchis retrobiloba n. sp. (Digenea: Gorgoderidae) from Astyanax fasciatus (Teleostei: Characidae) from southern Uruguay, with an emended diagnosis of the genus Dendrorchis. Journal of Parasitology, 91(5), 1204-1207.
Wallace, J.B., y Webster, J.R. (1996). The role of macroinvertebrates in stream ecosystem function. Annual review of entomology, 41(1), 115-139.

Williams, P., Biggs, J., Fox, G., Nicolet, P., y Whitfield, M. (2001). History, origins and importance of temporary ponds. En: Freshwater Forum, 17, $7-15$.

Editor de Sección: Carlos Iglesias 